\title{
Construction and implications of structural equation modeling network for pediatric cataract: a data mining research of rare diseases
}

\author{
Erping Long ${ }^{1}$, Shuangjuan $\mathrm{Xu}^{2}$, Zhenzhen Liu', Xiaohang $\mathrm{Wu}^{1}$, Xiayin Zhang ${ }^{1}$, Jinghui Wang ${ }^{1}$, Wangting Li ${ }^{1}$, \\ Runzhong Liư ${ }^{3}$, Zicong Chen ${ }^{3}$, Kexin Chen ${ }^{1}$, Tongyong $\mathrm{Yu}^{1}$, Dongxuan $\mathrm{Wu}^{1}$, Xutu Zhao ${ }^{1}$, Jingjing Chen ${ }^{1}$, \\ Zhuoling Lin ${ }^{1}$, Qianzhong Cao ${ }^{1}$, Duoru Lin ${ }^{1}$, Xiaoyan Li', Jingheng Cai ${ }^{2^{*}}$ and Haotian Lin ${ }^{{ }^{*}}$
}

\begin{abstract}
Background: The majority of rare diseases are complex diseases caused by a combination of multiple morbigenous factors. However, uncovering the complex etiology and pathogenesis of rare diseases is difficult due to limited clinical resources and conventional statistical methods. This study aims to investigate the interrelationship and the effectiveness of potential factors of pediatric cataract, for the exploration of data mining strategy in the scenarios of rare diseases.

Methods: We established a pilot rare disease specialized care center to systematically record all information and the entire treatment process of pediatric cataract patients. These clinical records contain the medical history, multiple structural indices, and comprehensive functional metrics. A two-layer structural equation model network was applied, and eight potential factors were filtered and included in the final modeling.

Results: Four risk factors (area, density, location, and abnormal pregnancy experience) and four beneficial factors (axis length, uncorrected visual acuity, intraocular pressure, and age at diagnosis) were identified. Quantifiable results suggested that abnormal pregnancy history may be the principle risk factor among medical history for pediatric cataracts. Moreover, axis length, density, uncorrected visual acuity and age at diagnosis served as the dominant factors and should be emphasized in regular clinical practice.
\end{abstract}

Conclusions: This study proposes a generalized evidence-based pattern for rare and complex disease data mining, provides new insights and clinical implications on pediatric cataract, and promotes rare-disease research and prevention to benefit patients.

Keywords: Rare diseases, Data mining, Pediatric cataract, Structural equation modeling

\section{Background}

Rare diseases are regarded as one of the main global disease burdens worldwide. Most of rare diseases are considered as complex diseases that are caused by a combination of multiple morbigenous factors [1]. However, uncovering the complex etiology and pathogenesis

\footnotetext{
* Correspondence: caijheng@mail.sysu.edu.cn; gddlht@aliyun.com

${ }^{2}$ School of Mathematics, Sun Yat-sen University, Guangzhou 510275, China ${ }^{1}$ State Key Laboratory of Ophthalmology, Zhongshan Ophthalmic Center, Sun Yat-sen University, Guangzhou 510060, China

Full list of author information is available at the end of the article
}

of rare diseases is difficult because of limited clinical resources and conventional statistical methods. Therefore, there is an urgent need to build a network combining multidimensional rare-disease data with innovative computational methods $[2,3]$.

Pediatric cataract is a typical rare disease with significant risk of visual loss [4]. Risk factors, systematic symptoms, and multi-dimension clinical evaluations are simultaneously indispensable for the pediatric cataract prevention and treatment process [5-9]. Therefore, pediatric cataract, which jointly combined the intricate 
pathogenesis and intractable clinical situation, is a suitable test case for the exploration of computational modeling and data mining for rare diseases.

Structural equation modeling (SEM) is a multivariate statistical technique that incorporates factors and path analysis [10], which is able to handle not only measurable variables, but also latent factors that cannot be measured or observed on their own. Latent variables, frequently encountered in substantive researches, are needed to be expressed by several measurable variables. However, most existing modeling techniques, such as multiple regression and observed variable analyses, cannot deal with latent variables whilst SEM compensates for these issues. Moreover, SEM can accurately measure unreliable events because it takes the measurement errors into account. Therefore, SEM has multiple advantages for the modeling of complex processes beyond simple correlations.

To explore the feasibility of applying SEM for the data mining of rare diseases, we established a pilot rare-disease specialized care center [Childhood Cataract Program of the Chinese Ministry of Health (CCPMOH)] to systematically record all basic information and the entire treatment process for pediatric cataract patients [11]. These clinical records contain the medical histories, multiple structural indices, and comprehensive functional metrics. We constructed a two-layer SEM network aiming to explore the interrelationship and the effectiveness of these potential factors and to provide clinical implications for pediatric cataract. We hope that this study will propose a generalized evidence-based pattern and valuable reference for rare and complex disease research.

\section{Methods}

The methods portion of the pipeline consists of three sections. The first section provides the procedure for the original data integration, description, and definition. The second section includes a detailed methodology for SEM network construction. The third section is concentrated on the network evaluation indices and contribution of factors, which could serves as a reference for clinical interpretation. Each section is described below. The study pipeline is presented in Fig. 1.

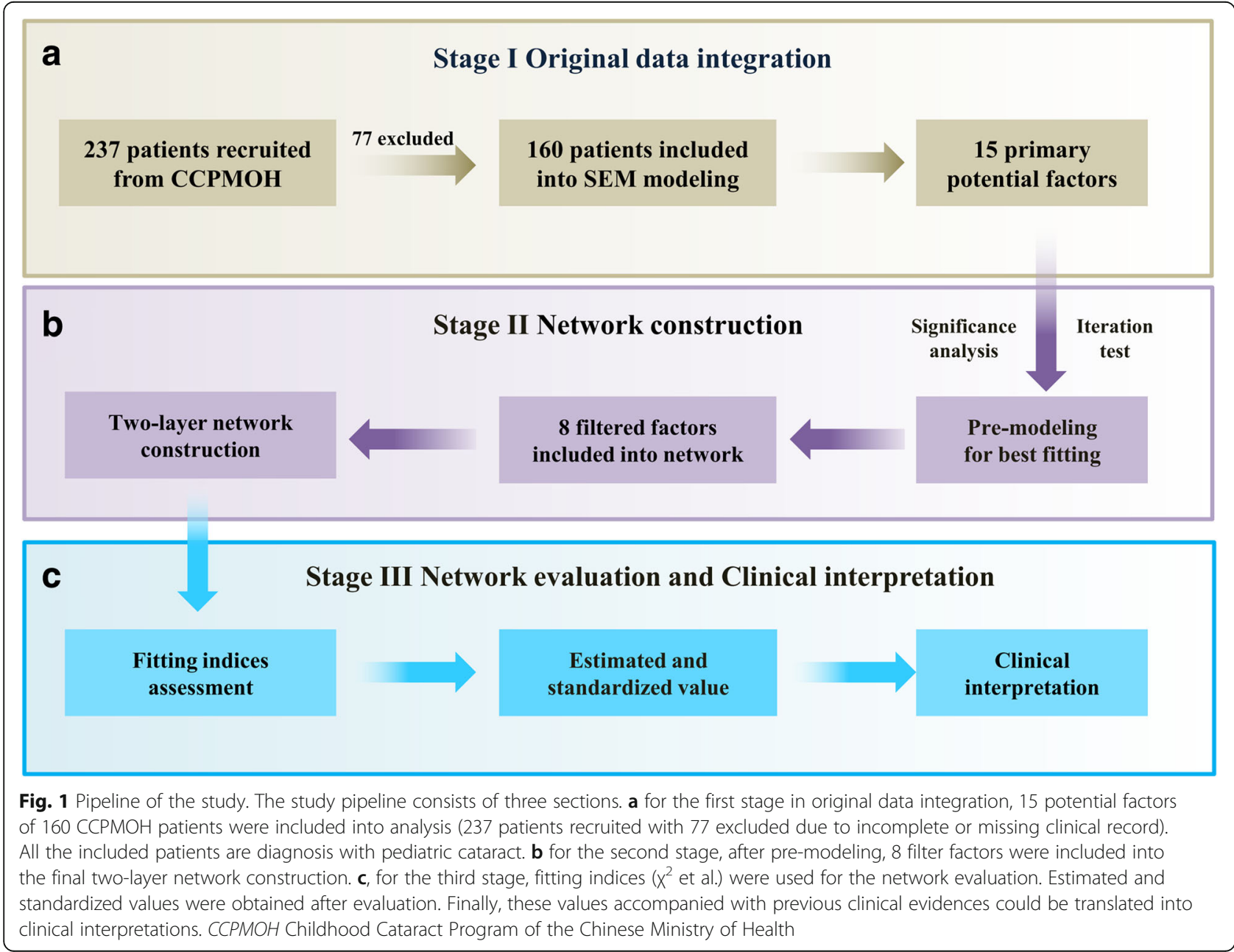




\section{Study population}

A total of 237 patients registered with the CCPMOH [11] were recruited from Zhongshan Ophthalmic Center [12], one of China's largest eye hospitals, located in Guangzhou city, south China. Seventy-seven patients with wide range of incomplete or missing clinical record were excluded. All the included patients are diagnosed with pediatric cataract according to the International Statistical Classification of Diseases and Related Health Problems 10th Revision (ICD-10) Version (Disease numbers: H26.0 and Q12.0) [12]. Finally, 160 patients' data records were included into the analysis and SEM construction. The dataset was anonymized throughout the research.

\section{Ethics approval}

The research protocol was approved by the Institutional Review Board/Ethics Committee of Sun Yat-sen University (Guangzhou, China). Informed written consent was obtained from at least one family member of each participating patient, and the tenets of the Declaration of Helsinki were followed throughout this study. To allow confidential evaluation of the use of a slit-lamp, a Tono-Pen, a Pentacam imaging system and the Teller visual acuity (VA) cards in this study, this trial was registered with the Clinical Research Internal Management System of CCPMOH. The authors affirm that all ongoing trials and trials related to this study are registered.

\section{Examination protocol and variable definitions}

All original variables and their SEM constructions are displayed in Table 1.

Table 1 Summary of original variables and SEM constructions

\begin{tabular}{lll}
\hline Two-layer Variables & One-layer Variables & Original Variables \\
\hline Overall index & Concomitant variables & Age at diagnosis \\
& Laterality \\
& Height \\
& Weight \\
& Family heredity history \\
& Abnormal parturition \\
& history \\
& Abnormal pregnant history \\
& AL \\
& Area \\
& Density \\
& Location \\
& Ocular complications \\
& IOP \\
& UCVA \\
& BCVA \\
\end{tabular}

AL Axial length, IOP Intraocular pressure, UCVA uncorrected visual acuity, BCVA best-corrected visual acuity
The age at diagnosis, height, weight, family hereditary history, abnormal parturition history, and abnormal pregnancy history were collected in regular clinical practice. The diagnosis of pediatric cataract was made by experienced ophthalmologists according to the ICD-10 Version [13]. The family hereditary history was defined as any similar disease history of immediate family members. The abnormal parturition history included but was not limited to an abnormal fetal position, abnormal placenta and amniotic fluid, fetal distress, breech birth, fetal macrosomia, and hypamnion. The abnormal pregnancy history included premature delivery, post-term pregnancy, pregnancy complicated with infection, gestational hypertension and gestational diabetes mellitus.

Area, density, and location are three critical lesion indices that are defined for the comprehensive evaluation and treatment decisions of pediatric cataracts (details definition in Additional file 1) [14].

Laterality was classified as bilateral cataracts or a unilateral cataract. For unilateral cataracts, data from the affected eye were included in the analysis and modeling. For bilateral cataracts, if the interocular lesion (area, density, and location) presented no differences, only the variables of the right eye were chosen for the modeling and analysis; otherwise, data from this patient were excluded to avoid bias caused by reduplicative datasets.

The ocular complications consisted of microphthalmia, micro- or megalocornea, keratoconus, glaucoma, traumatic or complicated cataracts, or vitreous and retinal diseases. Otherwise, the data were regarded as "no ocular complications".

The intraocular pressure (IOP) measurement was conducted using a Tono-pen tonometer (Reichert Inc., Seefeld, Germany) (details in Additional file 1) [15, 16].

\section{Axial length ( $A L)$}

The contact A scan (B-SCAN-Vplus/BIOVISION, Quantel Medical, Clermont-Ferrand, France) was used to obtain the AL measurements (details in Additional file 1).

\section{$V A$ - Uncorrected visual acuity (UCVA) and best-corrected visual acuity (BCVA)}

For preverbal children, a complete set of Teller VA Cards (Stereo Optical Company, Inc., IL, USA) was used to measure the monocular grating acuity of the eyes with cataracts [17]. The set consisted of 15 cards with gratings ranging in spatial frequencies from 0.32 cycles $/ \mathrm{cm}$ to 38 cycles $/ \mathrm{cm}$ in half-octave steps as well as a low vision card and a blank gray card. The infant was assessed using the standard procedure of the operation manual $[18,19]$. For verbal children, visual functions were measured and recorded using a LEA Symbols 13-Line Translucent Distance Chart (GoodLite Co., IL, USA) according to the standard procedure. 
The BCVA was assessed using spectacles after the UCVA examination. The results were translated into log MAR VA for analysis and modeling [20].

\section{Quality control}

Three experienced pediatric ophthalmologists (H.T.L., J.J.C. and Z.L.L.) performed all examinations according to our study protocols.

\section{Structural equation modeling}

For the pre-modeling, the age at diagnosis, height, weight, family hereditary history, abnormal parturition history, and abnormal pregnancy history were considered the concomitant variables. The AL, area, density, and location were loaded into the one-layer latent variance and termed the structural index (STR). The ocular complications, IOP, UCVA and BCVA were loaded into another one-layer latent variance and termed the functional index (FUN). The overall index (OVE) was settled as the two-layer latent variance. The aforementioned structure for premodeling is presented in Table 1.

In the pre-modeling process, primary significance analysis and iteration testing were applied for variable fitting. The combination of the variables for the best SEM fitting will be accept and proceeded into further analysis. After pre-modeling, a total of 8 filtered variables were selected and included in the final SEM (Table 2).

Table 2 Summary of the distribution of the 8 filtered variables included in the final SEM analysis

\begin{tabular}{|c|c|c|c|}
\hline $\begin{array}{l}\text { Two-layer } \\
\text { Variables } \\
\end{array}$ & $\begin{array}{l}\text { One-layer } \\
\text { Variables }\end{array}$ & Original Variables & Detailed distribution \\
\hline \multirow{12}{*}{$\begin{array}{l}\text { Overall } \\
\text { index }\end{array}$} & \multirow{3}{*}{$\begin{array}{l}\text { Concomitant } \\
\text { variables }\end{array}$} & Age at diagnosis & $50.99 \pm 36.38$ months \\
\hline & & \multirow{2}{*}{$\begin{array}{l}\text { Abnormal pregnancy } \\
\text { history }\end{array}$} & $23.13 \%(+)$ \\
\hline & & & $76.87 \%(-)$ \\
\hline & \multirow{7}{*}{$\begin{array}{l}\text { Structural } \\
\text { indices }\end{array}$} & $A L$ & $21.75 \pm 2.06 \mathrm{~mm}$ \\
\hline & & \multirow[t]{2}{*}{ Area } & 55\% (Extensive) \\
\hline & & & 45\% (Limited) \\
\hline & & \multirow[t]{2}{*}{ Density } & 37.5\% (Dense) \\
\hline & & & 63.5\% (Non-dense) \\
\hline & & \multirow[t]{2}{*}{ Location } & $60.63 \%$ (Central) \\
\hline & & & 39.37\% (Peripheral) \\
\hline & \multirow{2}{*}{$\begin{array}{l}\text { Functional } \\
\text { indices }\end{array}$} & $\mathrm{IOP}$ & $15.12 \pm 6.88 \mathrm{mmHg}$ \\
\hline & & UCVA & $0.77 \pm 0.44$ (logMAR) \\
\hline
\end{tabular}

A total of 160 participants from the CCPMOH database and 8 filtered variables were included in the final SEM analysis. The mean age of the included participants was 50.99 months \pm 36.38 months, and $23.13 \%(n=37)$ of our patients had an abnormal pregnancy history. For the STR network, the mean value of AL was $21.75 \mathrm{~mm} \pm 2.06 \mathrm{~mm} ; 55 \%(n=88)$ of our patients had an extensive area; $37.5 \%(n=60)$ of our patients had dense opacity, and $60.63 \%$ $(n=97)$ of our patients had opacity at central location. For the FUN network, the mean IOP value was $15.12 \mathrm{mmHg} \pm 6.88 \mathrm{mmHg}$, and the mean UCVA value (logMAR) was $0.77 \pm 0.44$

AL Axial length, IOP Intraocular pressure, UCVA uncorrected visual acuity
SEM was conducted to statistically test the interrelationships of the constructs and their relationships with the structural, functional, and overall components in our study population. Prior to modeling the relationship between latent variables, a measurement model was evaluated for each dietary behavior component. This step involves a confirmatory factor analysis to reveal the relationship between the latent variables and their indicator variables. The following step (i.e., testing of the structural model) estimates the strength of the relationships between these latent variables. It also allows for an examination of the direct and indirect effects among the constructs in the model. The data were examined prior to modeling to ensure that they met the assumptions of the proposed SEM and were analyzed using the robust weighted least squares procedure in Mplus 7.0 (Mplus, Los Angeles, Calif) [21].

To evaluate the goodness of fit of the model, the $x^{2}$ value was calculated together with the degrees of freedom and four other indices as follows: the root mean square error of approximation (RMSEA), the TuckerLewis index (TLI), the comparative fit index (CFI), and the weighted residual root mean square residual (WRMR). Values below 0.08 for RMSEA, below 1.0 for WRMR and above 0.90 for TFI and CFI indicate an acceptable fit of the data to the hypothesized model (details in Additional file 1).

\section{Results}

\section{Participant characteristics}

A total of 160 participants from the CCPMOH database with 8 filtered variables were included in the final SEM analysis (details in Table 2). The mean age of the included participants was 50.99 months \pm 36.38 months and $23.13 \%(n=37)$ of our patients had an abnormal pregnancy history. For the STR network, the mean AL value was $21.75 \mathrm{~mm} \pm 2.06 \mathrm{~mm}, 55 \%(n=88)$ of our patients had an extensive area, 37.5\% $(n=60)$ of our patients had dense opacity, and $60.63 \%(n=97)$ of our patients' opacities had central locations. For the FUN network, the mean IOP value was $15.12 \pm 6.88 \mathrm{mmHg}$, and the mean UCVA value (logMAR) was $0.77 \pm 0.44$.

\section{Goodness-of-fit of the models}

As shown in Table 3, the overall fit of the measurement and the structural models was satisfactory $\left(X^{2}=26.093\right.$, $d f=16, P=0.1113$; RMSEA $=0.053, P=0.422$; CFI $=0.995$; TFI $=0.992 ;$ WRMR $=0.781)$. Finally, the estimates of the SEM constructs are presented in Table 4.

\section{Interrelationships of the constructs}

The standardized SEM architecture and interrelationships of the constructs are displayed in Fig. 2. 
Table 3 Summary of statistics of the goodness-of-fit indices

\begin{tabular}{ll}
\hline Goodness-of-fit index & Results \\
\hline$X^{2}$ (Chi-square value) & $X^{2}=26.093, d f=16, P=0.1113$ \\
RMSEA & $0.053, P=0.422$ \\
CFI & 0.995 \\
TFI & 0.992 \\
WRMR & 0.781
\end{tabular}

The overall fit of the measurement and the structural models was satisfactory $\left(X^{2}=26.093, d f=16, P=0.1113 ;\right.$ RMSEA $=0.053, P=0.422 ; C F I=0.995$;

$\mathrm{TFI}=0.992 ; \mathrm{WRMR}=0.781$ )

RMSEA Root mean square error of approximation, TLI Tucker- Lewis index, CFI Comparative fit index, WRMR Weighted residual root mean square residual

\section{STR networks}

The lesion area $(-0.243)$, density $(-0.57)$, and location $(-0.357)$ simultaneously had negative correlations with the STR index. The AL variable had a positive correlation with the STR index (0.615).

\section{FUN networks}

The presence of higher UCVA was positively associated with a higher FUN index (0.571), suggesting that patients with higher UCVA will accordingly have better visual functions. Furthermore, the IOP value was positively correlated with the FUN index (0.342). Because the main range of the IOP is below $21 \mathrm{mmHg}$ (normal range), this result mainly reveals the functional support role of IOP, which is necessary for visual maintenance.

\section{Overall networks}

A positive relationship in the model was the link among the FUN (0.974) and STR indices (1.046) and the overall network index, which indicates that function and structure are two of the main dimensions for overall disease evaluation, with structure forming the base of visual function (0.052). The age at diagnosis (0.753) was positively correlated with the overall index, whereas an abnormal pregnancy experience
$(-0.26)$ had a negative relationship with the overall index. These results demonstrate that age is an important indicator for the visual maturation and that an abnormal pregnancy experience will have a detrimental influence on overall visual function.

\section{Clinical interpretations}

The results from SEM network quantifiably illuminate the effect of eight potential factors (four risk factors: area, density, location, and abnormal pregnancy experience; four beneficial factors: AL, UCVA, IOP value, and age at diagnosis). For structural effect, extensive lesion area, dense opacity and central location will present increasing severity of cataract but AL growth will be a positive indicator for the better structural maturity. Furthermore, both UCVA and IOP value in normal range presented beneficial functional effect and could be the positive evaluation indicators for patients. Additionally, patients with earlier diagnosis age and abnormal pregnancy experience will be the indicators for a more severe status. All these identified factors are also valuable references and indicators for patients' prognoses.

\section{Discussion}

It is imperative to study rare and complex diseases in a systematic manner

Studying rare and complex disease is a great challenge for researchers and medical practitioners [22]. Rare and complex diseases tend to be caused by a system, with interconnected entities being modeled as nodes and their connections as edges to comprise an intricate pathogenic network [23]. Therefore, it is always difficult for researchers to explicitly elucidate the principle nosogenesis, individual predisposition and related risk factors.

With the advance and popularization of medical detecting and monitoring equipment, medical practitioners should address an increasing number of

Table 4 Modeling estimation indices of the SEM constructs

\begin{tabular}{|c|c|c|c|c|c|}
\hline Variables & & Coefficient estimates value & Standardized coefficient estimation & Standard error & $P$ value \\
\hline \multirow[t]{4}{*}{ Structural indices } & $\mathrm{AL}$ & 1.000 & 0.615 & - & - \\
\hline & Area & -0.203 & -0.243 & 0.092 & 0.027 \\
\hline & Density & -0.543 & -0.570 & 0.106 & 0.000 \\
\hline & Location & -0.308 & -0.357 & 0.107 & 0.004 \\
\hline \multirow[t]{2}{*}{ Functional indices } & $\mathrm{IOP}$ & 1.000 & 0.342 & - & - \\
\hline & UCVA & 0.054 & 0.571 & 0.033 & 0.098 \\
\hline \multirow[t]{2}{*}{ Overall index by } & STRI & 1.000 & 1.046 & - & - \\
\hline & FUNI & 1.785 & 0.974 & 0.493 & 0.000 \\
\hline \multirow[t]{2}{*}{ Overall index on } & Age at diagnosis & 0.027 & 0.753 & 0.004 & 0.000 \\
\hline & Abnormal pregnancy history & -0.787 & -0.260 & 0.266 & 0.003 \\
\hline
\end{tabular}

AL Axial length, IOP Intraocular pressure, UCVA uncorrected visual acuity, STRI Structural indices, FUNI Functional indices 


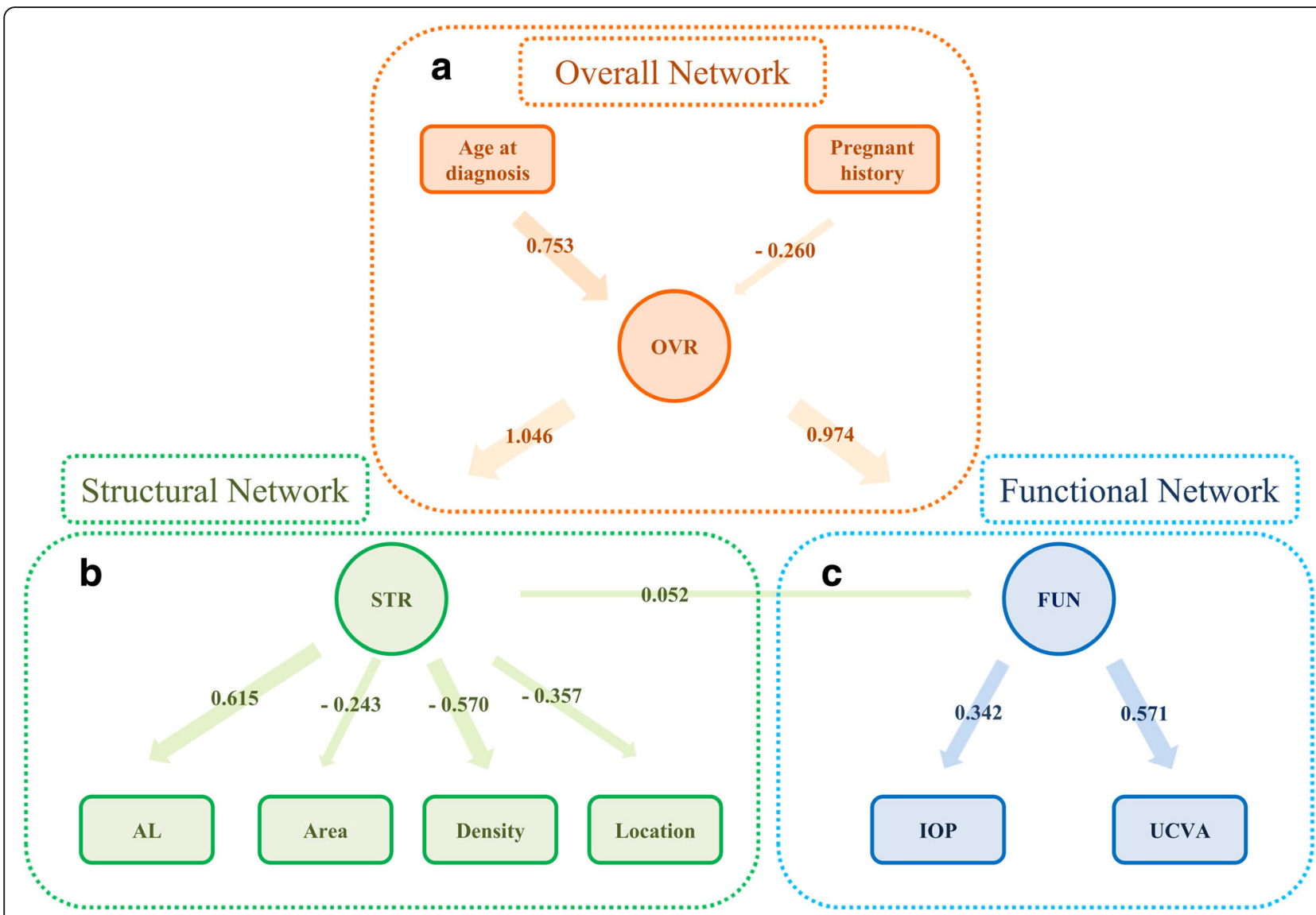

Fig. 2 The standardized SEM architecture and interrelationships of the constructs. a The overall network exhibited a positive correlation with the FUN (0.974) and STR indices (1.046). The age at diagnosis (0.753) was positively correlated with the overall index, while an abnormal pregnancy experience $(-0.26)$ exhibited a negative relationship with the overall index. b The lesion area $(-0.243)$, density $(-0.57)$ and location $(-0.357)$ were all negatively correlated with the STR index. Meanwhile, the AL variable has the positive correlation with the STR index (0.615). c, Increased UCVA was directly associated with a higher FUN index (0.571), and the IOP value was positively correlated with the FUN index (0.342). AL Axial length, IOP Intraocular pressure, UCVA uncorrected visual acuity, BCVA best-corrected visual acuity, STR Structural indices, FUN Functional indices, OVE Overall indices

clinical indices. However, quite a few examinations or clinical indices seem to be unnecessary or to provide ambiguous information for the diagnosis and the treatment decision, which is contrary to the goals of precision medicine $[24,25]$. Therefore, it is imperative to uncover the interrelationship and the effectiveness of these potential clinical indices and risk factors and to integrate these elements to construct a holistic network capable of providing insights into rare disease evidence-based prevention and treatment.

To obtain sufficient evidence and data for rare diseases, we established the $\mathrm{CCPMOH}$ program and built a rare disease data integration platform [11]. Medical history, multiple structural indices, and comprehensive functional metrics were collected for the primary investigation. After pre-modeling, a total of 8 filtered variables were selected and included in the final SEM modeling.
Implications from filtered variables: abnormal pregnancy history may be the principle risk factor among medical history for pediatric cataracts

Rare diseases, including pediatric cataracts, are always associated with birth defects [26]. However, pediatric cataracts present great genetic heterogeneity, and a considerable proportion of pediatric cataracts should be considered in the context of interactions among the environment and heredity [27]. Among the 8 filtered variables, abnormal pregnancy history was included but family hereditary history and abnormal parturition history were excluded from the final network. In addition to the final network contribution, these results indicate that abnormal pregnancy history may be the principle risk factor for pediatric cataracts. Previous studies reported that pediatric cataracts had an association with rubella infection $[28,29]$, which was in agreement with our findings on the abnormal pregnancy history. Therefore, more attentions 
should be focused on patients with abnormal pregnancy history in screening and early diagnostics of pediatric cataract.

\section{Our results are concordant with conventional medical knowledge and validate the reliability of the SEM network}

To systematically model our clinical indices, we classified our records into two separated latent variances (structural indices or function indices) for two-layer SEM construction. Two essential steps are needed for the SEM clinical interpretation. First, we should holistically evaluate the SEM network using existing evidence and the clinical consensus. Our results indicate that the functional and structural networks have positive support from the overall network and that the structural network has a positive supporting effect on the functional network. Additionally, the lesion area, density and location have a negative effect on the structural network, and UCVA is a positive indicator for the functional network. These results are concordant with conventional medical knowledge and validate the reliability of the SEM network.

Implications from SEM network index: the risk or beneficial effects of the potential factors are confirmed to provide insights into rare disease prevention and treatment

After verifying the reliability of the model in the preliminary test, the second step is to explain the model and provide novel and valuable clinical information. For the structural network, AL presents a positive effect in our study population. The main study population was younger than 2 years of age; thus, in the early stage, increasing AL is a beneficial indictor for eyeball development instead of myopia progression. For the functional network, the IOP plays as a supporting role, which reveals that an IOP in the normal range (the main range of our IOP is below $21 \mathrm{mmHg}$ ) is not a risk factor for glaucoma but instead is a supporting factor for visual function and a positive nutrient media for the functional component. For the overall network, age at diagnosis is also a positive indicator, which demonstrates that the severe pediatric cataract is more likely to be diagnosed in the early stage. These implications could be an important supplement to the mainstream theory of pediatric cataract.

Implications from weight of index: AL, density, UCVA and age at diagnosis served as the dominant factors and might be emphasized in our regular clinical practice

The weight of each index is also valuable information for the clinical interpretation. For the structural network, AL and density are the dominant factors and can be considered key indicators for severity and prognosis. For the functional network, UCVA plays the main role along with the subordinate beneficial effect from the IOP. The functional and structural networks almost equally provide a contribution to the overall network. Furthermore, the age at diagnosis is more crucial than an abnormal pregnancy experience in the overall network. Therefore, AL, density, UCVA and age at diagnosis might be emphasized in our regular clinical practice.

\section{Acceptable fitting of SEM network and study limitation}

We performed the goodness-of-fit validation to ensure the meaning and reliability of the SEM network. The key indicators, $X^{2}$-values and RMSEA indicated an acceptable fit of the data to our model along with the TFI, CFI, and WRMR, which supported the same conclusion. Although the modeling reliability of our variables was not sufficiently ideal to show great explanation power, the overall fitting of the SEM network is acceptable.

The main limitation of our study is the data quality. Two main effects (missing data and inevitable errors) caused by data quality are introduced as follows. Missing data will directly influence the model fitting; thus, the reason for the exclusion of the primary indices should not be merely interpreted by their limited or subordinate effect on disease. Notably, some valuable indices might be excluded by the effect of the missing data. Moreover, the missing data could be a possible explanation for the unsatisfactory modeling reliability. Additionally, uncooperative measurement is common for our regular examination procedure because the majority of our patients are under 2 years of age, which causes fairly inevitable measuring errors. Furthermore, the VA evaluation is a somewhat subjective measurement and the medical history is a retrospective inquiry, which might present less accuracy. Our future study aims are to include a more dimensional and more complete rare disease dataset with a larger sample size for modeling and data mining.

\section{Conclusions}

In conclusion, our study proposed a feasible pattern for data mining using a rare disease dataset. We integrated the multidimensional dataset and constructed a twolayer SEM network to identify valuable clinical indices and uncover the interrelationships and the effectiveness of these potential factors. Four risk factors and four beneficial factors were filtered and confirmed with reliable clinical implications. This research could promote rare disease research and prevention to substantially benefit rare disease patients.

\section{Additional file}

Additional file 1: The detailed information for potential variables and evaluation indices are presented. (DOCX $16 \mathrm{~kb}$ ) 


\section{Abbreviations}

AL: Axial length; BCVA: Best-corrected visual acuity; CCPMOH: Childhood cataract program of the chinese ministry of health; CFI: Comparative fit index; FUN: Functional index; ICD-10: International statistical classification of diseases and related health problems 10th revision; IOL: Intraocular lens; IOP: Intraocular pressure; OVE: Overall index; RMSEA: Root mean square error of approximation; SEM: Structural equation modeling; STR: Structural index; TLI: Tucker- lewis index; UCVA: Uncorrected visual acuity; VA: Visual acuity; WRMR: Weighted residual root mean square residual

\section{Acknowledgements}

We thank all the members in the CCPMOH. CCPMOH Study Group has been founded and developed in Zhongshan Ophthalmic Center, Sun Yat-sen University since 2010 because of the Childhood Cataract Program of the Chinese Ministry of Health.

\section{Consent for publication}

Not applicable.

\section{Funding}

This study was supported by NSFC (91546101), the Guangdong Provincial Natural Science Foundation for Distinguished Young Scholars of China (2014A030306030), the Youth Science and Technology Innovation Talents Funds in a Special Support Plan for High Level Talents in Guangdong Province (2014TQ01R573), Guangdong Province Universities and Colleges Youth Pearl River Scholar Funded Scheme (2016), and the high-performance grid computing platform of Sun Yat-sen University. The funders had no role in study design, data collection and analysis, decision to publish, or preparation of the manuscript.

\section{Availability of data and materials}

The datasets of the current study are available from the corresponding author on reasonable request.

\section{Authors' contributions}

Concept and design (HTL, EPL and JHC); Data acquisition, statistical analysis, and interpretation (EPL, SJX, ZZL, XHW, XYZ, JHW, WTL, RZL, ZCC, KXC, TYY, DXW, XTZ, JJC, ZLL, QZC, DRL, XYL and HTL); Drafting of the manuscript (EPL and HTL); Critical revision of the manuscript (SJX, ZZL, XHW, XYZ, JHW, WTL, RZL, ZCC, KXC, TYY, DXW, XTZ, JJC, ZLL, QZC, DRL, XYL and JHC); All authors gave the final approval of the manuscript and agreed to be accountable for all aspects of the work in ensuring that questions related to the accuracy or integrity of any part of the work are appropriately investigated and resolved.

\section{Competing interests}

The authors declare that they have no competing interests.

\section{Ethics approval and consent to participate}

All study procedures were performed in accordance with the tenets of the Declaration of Helsinki, and the study was approved by the Ethics Committee of the Zhongshan Ophthalmic Center. Written informed consent was obtained from all the study participants' parents or legal guardian.

\section{Publisher's Note}

Springer Nature remains neutral with regard to jurisdictional claims in published maps and institutional affiliations.

\section{Author details}

'State Key Laboratory of Ophthalmology, Zhongshan Ophthalmic Center, Sun Yat-sen University, Guangzhou 510060, China. ${ }^{2}$ School of Mathematics, Sun Yat-sen University, Guangzhou 510275, China. ${ }^{3}$ School of Public health, Sun Yat-sen University, Guangzhou 510080, China.

\section{Received: 27 February 2017 Accepted: 15 May 2017}

\section{Published online: 19 May 2017}

\section{References}

1. Wu Z. Challenges and opportunities in the studies on rare diseases. Transl Pediatr. 2015;4(3):239.

2. Remuzzi G, Garattini S. Rare diseases: what's next. Lancet. 2008: 371(9629):1978-9.
3. Lakdawala NK. Big data for a rare disease: examining heart transplantation for left ventricular noncompaction in the united network of organ sharing registry. J Heart Lung Transplant. 2015;34(6):759-60.

4. Medsinge A, Nischal KK. Pediatric cataract: challenges and future directions. Clin Ophthalmol. 2015;9:77-90.

5. Patil-Chhablani P, Kekunnaya R, Nischal KK. Complex cases in pediatric cataract. Dev Ophthalmol. 2016;57:85-106.

6. Lambert SR. Changes in ocular growth after pediatric cataract surgery. Dev Ophthalmol. 2016;57:29-39.

7. Song Z, Zhao D, LV C, Pu W, Xiao W. Ten-year etiologic review of Chinese children hospitalized for pediatric cataracts. Eye Sci. 2014; 29(3):138-42.

8. Gillespie RL, Urquhart J, Anderson B, Williams S, Waller S, Ashworth J, Biswas $\mathrm{S}$, Jones $\mathrm{S}$, Stewart F, Lloyd IC, et al. Next-generation sequencing in the diagnosis of metabolic disease marked by pediatric cataract. Ophthalmology. 2016;123(1):217-20.

9. Long E, Chen J, Liu Z, Lin Z, Cao Q, Zhang X, Li X, Luo L, Lin H, Chen $W$, et al. Interocular anatomical and visual functional differences in pediatric patients with unilateral cataracts. BMC Ophthalmol. 2016; 16(1):192.

10. Kline RB. Latent variable path analysis in clinical research: a beginner's tour guide. J Clin Psychol. 1991:47(4):471-84.

11. Lin H, Long E, Chen W, Liu Y. Documenting rare disease data in China. Science. 2015:349(6252):1064

12. Dolgin E. The myopia boom. Nature. 2015;519(7543):276-8.

13. WHO. The International Classification of Diseases. 2010. http://www.who.int/ classifications/icd/en. Accessed 16 Apr 2016.

14. Long $E$, Lin H, Liu Z, Wu X, Wang L, Jiang J, An Y, Lin Z, Li X, Chen J, et al. An artificial intelligence platform for the multihospital collaborative management of congenital cataracts. Nature biomedical engineering. 2017;0024(1):1-8.

15. Lin H, Chen W, Luo L, Zhang X, Chen J, Lin Z, Qu B, Zhan J, Zheng D, Zhong $X$, et al. Ocular hypertension after pediatric cataract surgery: baseline characteristics and first-year report. PLoS One. 2013;8(7):e69867.

16. Jonas JB, Degenring RF, Kreissig I, Akkoyun I, Kamppeter BA. Intraocular pressure elevation after intravitreal triamcinolone acetonide injection. Ophthalmology. 2005:112(4):593-8.

17. Mayer DL, Beiser AS, Warner AF, Pratt EM, Raye KN, Lang JM. Monocular acuity norms for the teller acuity cards between ages one month and four years. Invest Ophthalmol Vis Sci. 1995;36(3):671-85.

18. Cavallini A, Fazzi E, Viviani V, Astori MG, Zaverio S, Bianchi PE, Lanzi G. Visual acuity in the first two years of life in healthy term newborns: an experience with the teller acuity cards. Funct Neurol. 2002;17(2):87-92.

19. Ciocler FP, Dantas PE. Assessment of visual acuity in patients with dementia using teller acuity cards. Strabismus. 2013;21(2):93-7.

20. Borisovsky G, Silberberg G, Wygnanski-Jaffe T, Spierer A. Results of congenital cataract surgery with and without intraocular lens implantation in infants and children. Graefes Arch Clin Exp Ophthalmol. 2013;251(9):2205-11.

21. Muthen L.K. MBO: Mplus user's guide. 7th edn. Muthen \& Muthen: Los Angeles, CA 1998-2012.

22. Schieppati A, Henter Jl, Daina E, Aperia A. Why rare diseases are an important medical and social issue. Lancet. 2008;371(9629):2039-41.

23. Gong S, Jin S. Current progress in the management of rare diseases and orphan drugs in China. Intractable Rare Dis Res. 2012:1(2):45-52.

24. Collins FS, Varmus $\mathrm{H}$. A new initiative on precision medicine. N Engl J Med. 2015:372(9):793-5.

25. Castaneda C, Nalley K, Mannion C, Bhattacharyya P, Blake P, Pecora A, Goy A, Suh KS. Clinical decision support systems for improving diagnostic accuracy and achieving precision medicine. J Clin Bioinforma. 2015:5:4.

26. Chinta S, Pehere N. Pediatric cataract. Ophthalmology. 2011;118(8):1692. author reply 1692

27. Gillespie RL, O'Sullivan J, Ashworth J, Bhaskar S, Williams S, Biswas S, Kehdi E, Ramsden SC, Clayton-Smith J, Black GC, et al. Personalized diagnosis and management of congenital cataract by next-generation sequencing. Ophthalmology. 2014;121(11):2124-2137.e1-2.

28. Eckstein MB, Brown DW, Foster A, Richards AF, Gilbert CE, Vijayalakshmi P. Congenital rubella in south India: diagnosis using saliva from infants with cataract. BMJ. 1996:312(7024):161

29. Khan NA, Kazzi SN. Yield and costs of screening growth-retarded infants for torch infections. Am J Perinatol. 2000;17(3):131-5. 